\title{
Fast ion D-alpha measurements using a bandpass-filtered system on EAST
}

Zhang, J.; Huang, J.; Chang, J. F.; Wu, C. R.; Heidbrink, W. W.; Salewski, M.; Madsen, B.; Zhu, Y. B.; von Hellermann, M. G.; Gao, W.

Total number of authors:

12

Published in:

Review of Scientific Instruments

Link to article, DOI:

$10.1063 / 1.5038828$

Publication date:

2018

Document Version

Publisher's PDF, also known as Version of record

Link back to DTU Orbit

Citation (APA):

Zhang, J., Huang, J., Chang, J. F., Wu, C. R., Heidbrink, W. W., Salewski, M., Madsen, B., Zhu, Y. B., von Hellermann, M. G., Gao, W., Xu, Ž., \& Wan, B. (2018). Fast ion D-alpha measurements using a bandpass-

filtered system on EAST. Review of Scientific Instruments, 89(10), [10D121]. https://doi.org/10.1063/1.5038828

\section{General rights}

Copyright and moral rights for the publications made accessible in the public portal are retained by the authors and/or other copyright owners and it is a condition of accessing publications that users recognise and abide by the legal requirements associated with these rights.

- Users may download and print one copy of any publication from the public portal for the purpose of private study or research.

- You may not further distribute the material or use it for any profit-making activity or commercial gain

- You may freely distribute the URL identifying the publication in the public portal 


\section{Fast ion D-alpha measurements using a bandpass-filtered system on EAST}

J. Zhang, J. Huang, J. F. Chang, C. R. Wu, W. W. Heidbrink, M. Salewski, B. Madsen, Y. B. Zhu, M. G. von Hellermann, W. Gao, Z. Xu, and B. Wan

Citation: Review of Scientific Instruments 89, 10D121 (2018); doi: 10.1063/1.5038828

View online: https://doi.org/10.1063/1.5038828

View Table of Contents: http://aip.scitation.org/toc/rsi/89/10

Published by the American Institute of Physics

\section{Articles you may be interested in}

Velocity-space sensitivities of neutron emission spectrometers at the tokamaks JET and ASDEX Upgrade in deuterium plasmas

Review of Scientific Instruments 88, 073506 (2017); 10.1063/1.4991651

Simultaneous measurement of C VI, Ne X, and Li III charge exchange lines on EAST

Review of Scientific Instruments 89, 10D119 (2018); 10.1063/1.5036835

Measurement of the toroidal radiation asymmetry during massive gas injection triggered disruptions on $\mathrm{J}$ TEXT

Review of Scientific Instruments 89, 10E113 (2018); 10.1063/1.5035187

An extended depth-of-field imaging system with a non-rotationally symmetric phase mask

Review of Scientific Instruments 89, 103101 (2018); 10.1063/1.5023292

Reconstruction of radiation profiles near the plasma boundary using an infrared imaging video bolometer in KSTAR

Review of Scientific Instruments 89, 10E111 (2018); 10.1063/1.5038904

Fast analysis of collective Thomson scattering spectra on Wendelstein 7-X

Review of Scientific Instruments 89, 083507 (2018); 10.1063/1.5035416

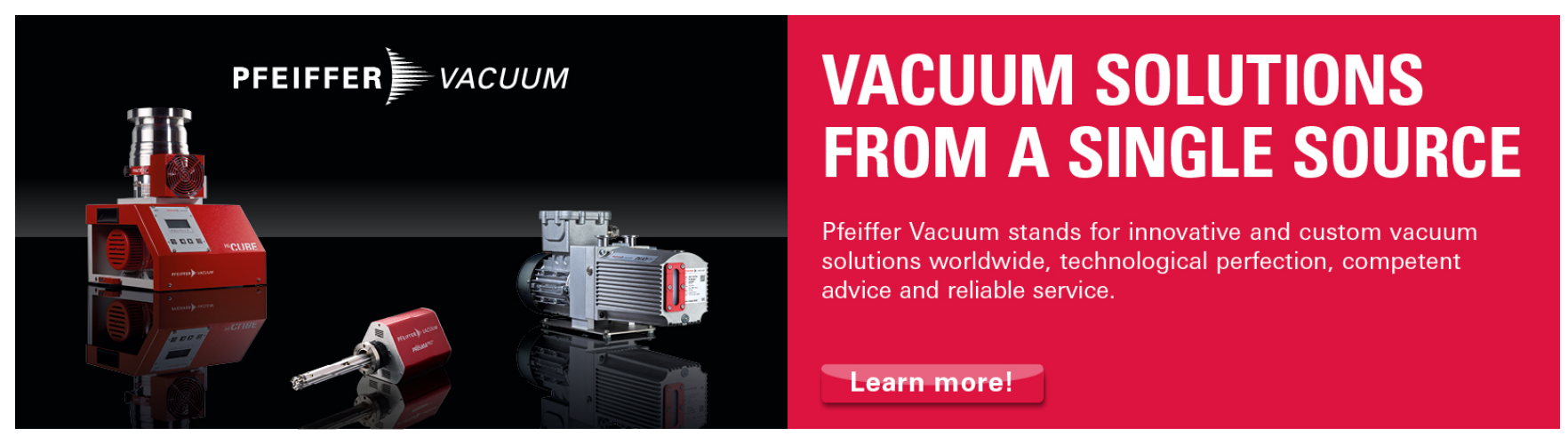




\title{
Fast ion D-alpha measurements using a bandpass-filtered system on EAST
}

\author{
J. Zhang, ${ }^{1,2}$ J. Huang, ${ }^{1}$ J. F. Chang, ${ }^{1, \text { a) }}$ C. R. Wu ${ }^{1,2}$ W. W. Heidbrink ${ }^{3}$ M. Salewski, ${ }^{4}$ \\ B. Madsen, ${ }^{4}$ Y. B. Zhu, ${ }^{3}$ M. G. von Hellermann, ${ }^{5}$ W. Gao, ${ }^{1}$ Z. Xu, ${ }^{6,7}$ and B. Wan ${ }^{1}$ \\ ${ }^{1}$ Institute of Plasma Physics, Chinese Academy of Sciences, P.O. Box 1126, 230031 Hefei, Anhui, China \\ ${ }^{2}$ University of Science and Technology of China, Hefei, Anhui 230026, China \\ ${ }^{3}$ Department of Physics and Astronomy, University of California, Irvine, California 92697, USA \\ ${ }^{4}$ Department of Physics, Technical University of Denmark, Kongens Lyngby, Denmark \\ ${ }^{5}$ FOM Institute DIFFER, Nieuwegein 3430 BE, The Netherlands \\ ${ }^{6}$ Advanced Energy Research Center, Shenzhen University, Shenzhen 518060, People's Republic of China \\ ${ }^{7}$ Key Laboratory of Optoelectronic Devices and Systems of Ministry of Education and Guangdong Province, \\ College of Optoelectronic Engineering, Shenzhen University, Shenzhen 518060, People's Republic of China
}

(Presented 19 April 2018; received 6 May 2018; accepted 15 June 2018; published online 4 October 2018)

\begin{abstract}
Based on the charge exchange reaction between fast ions and a neutral beam, fast ion features can be inferred from the spectrum of Doppler-shifted Balmer-alpha light from energetic hydrogenic atoms. In order to study the interaction between instabilities and fast-ion transport, recently we extended the fast ion D-alpha (FIDA) measurements by using a combination of a bandpass filter and a photomultiplier tube (PMT) (f-FIDA). A bandpass filter selects the desired spectral band from $651 \mathrm{~nm}$ to $654 \mathrm{~nm}$ before detection by the PMT. Preliminary data from the EAST tokamak show that the active signals have been detected from reneutralized beam ions along the vertical and tangential viewing geometries. The details will be presented in this paper to primarily address the specifications and performance of f-FIDA hardware components and preliminary FIDA measurements. Published by AIP Publishing. https://doi.org/10.1063/1.5038828
\end{abstract}

\section{INTRODUCTION}

The research on fast ions in toroidal magnetic fusion devices becomes more important in recent years because with the promotion of the plasma parameters and the heating methods, such as neutral beam injection (NBI) and ion cyclotron acceleration (ICRH), the fast ion product and its impact on plasma become non-negligible. Several diagnostics of fast ions have been developed on fusion devices. The fast-ion D-alpha diagnostic (FIDA) ${ }^{1}$ first commissioned on DIII- $\mathrm{D}^{2}$ in 2004 , is based on the charge exchange reaction between fast ions and a neutral beam, and it is well known that fast ion features can be inferred from the Dopplershift of Balmer-alpha light from energetic hydrogen (deuterium) atoms. Then it has been applied on many magnetic devices. ${ }^{3-10}$

To investigate the behavior of fast ions on EAST (Experimental Advanced Superconducting Tokamak), a FIDA diagnostic system with a spectrometer (s-FIDA) ${ }^{11}$ was mounted since 2014 which is mainly used to analyze the different energy components of fast ions. In order to study the interaction between instabilities and fast-ion transport, recently the FIDA system was extended by using a combination of a bandpass filter and a photomultiplier tube (PMT) (f-FIDA), which was first commissioned on NSTX $^{3}$ and also applied on DIII-D, ${ }^{4}$ and it will provide the temporal evolution of the blue shifted component of the Doppler shifted spectrum by integrating the

Note: Paper published as part of the Proceedings of the 22nd Topical Conference on High-Temperature Plasma Diagnostics, San Diego, California, April 2018.

a) Author to whom correspondence should be addressed: changjiaf@ipp.ac.cn desired spectral band from $651 \mathrm{~nm}$ to $654 \mathrm{~nm}$ before detection by the PMT.

This paper is structured as follows. Section II introduces the current FIDA diagnostic layout on EAST. The instrument performance of the new f-FIDA systems including a comparison between multi-channel f-FIDA with array-PMT and single-PMT is shown in Sec. III. In Sec. IV, the application of FIDA in L-mode MHD quiescent plasmas is presented. Section V presents a summary.

\section{FIDA DIAGNOSTIC LAYOUT}

Figure 1 shows the FIDA diagnostic layout on EAST. ${ }^{11}$ The new f-FIDA systems are shown in the diagnostic room part of Fig. 1. EAST has both co-current (named as NBI1R, NBI1L) and counter-current (named as NBI2R, NBI2L) neutral beam injection (NBI). Each has two sources which can deliver 2-4 MW beam power with 50-80 keV beam energy, as shown in the EAST hall part of Fig. 1. Now, the diagnostic beam of the FIDA system is the beam of NBI1L. The active viewers including tangential and vertical views that intersect the $\mathrm{NB}$ are toward the $\mathrm{O}$ horizontal and $\mathrm{B}$ vertical ports, covering the region of major radii from $177 \mathrm{~cm}$ to $237 \mathrm{~cm}$ and from $177 \mathrm{~cm}$ to $205 \mathrm{~cm}$, respectively. The passive vertical $\mathrm{N}$-port view that do not intersect a NB are geometrically the same as the active vertical B-port view. The optical fibers guide the signals to the FIDA instruments in the FIDA diagnostic room, keeping the detectors away from neutrons and gamma emission. The patch panels of the optical fiber link the fibers to the instruments allowing a flexible change of observation ports and instruments. To obtain good 


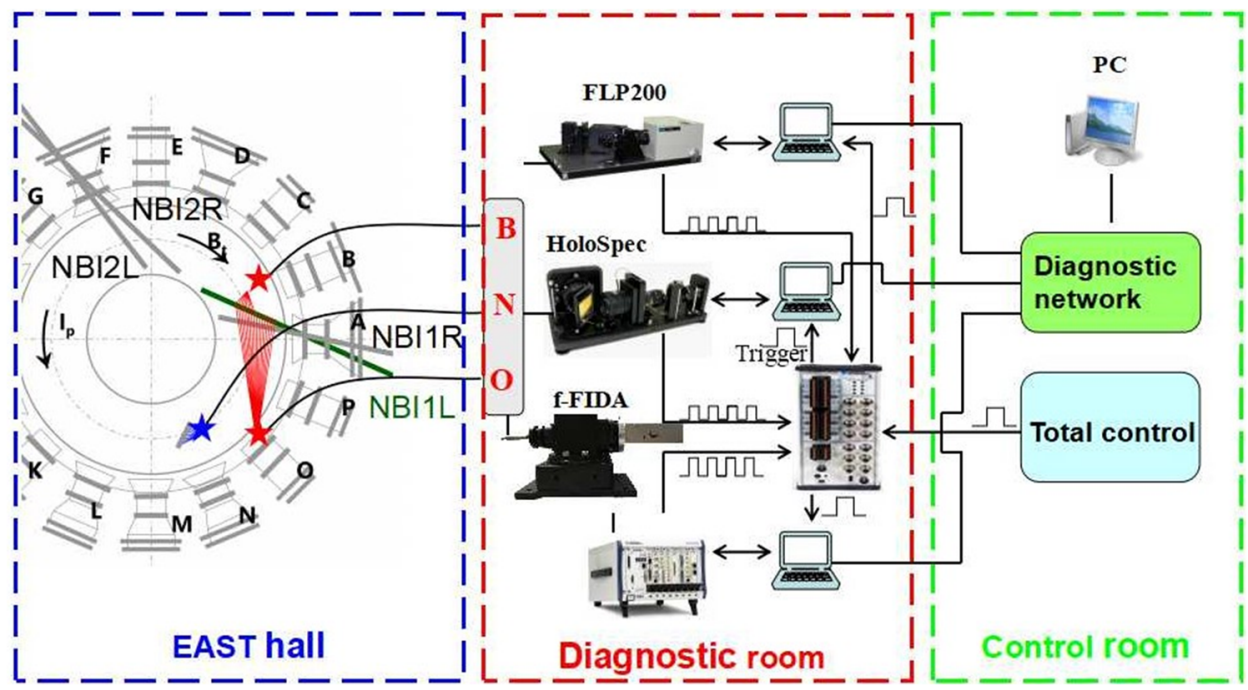

FIG. 1. The FIDA diagnostic layout on EAST.

spectral resolution and high temporal resolution, respectively, high resolution spectrometers are equipped with both FLP200 and HoleSpec spectrometers, ${ }^{11}$ and fast filter-based photomultiplier systems including the array-PMT and single-PMT have been both available for the complementary measurements.

\section{INSTRUMENT PERFORMANCE OF f-FIDA SYSTEMS}

\section{A. f-FIDA system with array-PMT}

The configuration of the f-FIDA system with an arrayPMT is illustrated in Fig. 2. Light from EAST is collected by an eight-fiber array, and each fiber has $1500 \mu \mathrm{m}$ core diameters and a numerical aperture of 0.37 , and the output spot of the fiber is $1.5 \mathrm{~mm}$ that is a surface light source. However, the input light has a certain divergence. The longer the focal length of the input lens, the smaller the divergence of the output light, but the larger the light spot. So when the optical fiber spot size is determined, the input lens is determined. The input lens (1) has a focal length of $10 \mathrm{~mm}$ and an outer diameter of $5 \mathrm{~mm}$, its material is quartz, and the divergence angle at this time is $5^{\circ}$. In order to make efficient use of the light energy and reduce cross-interference, the OD6 bandpass filters (2) with a center

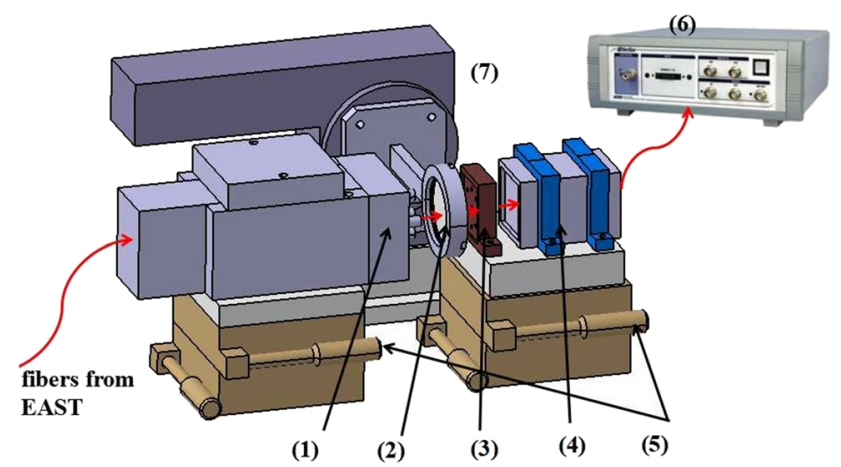

FIG. 2. The schematic of the multi-channel f-FIDA with array-PMT. (1) Input lens; (2) bandpass filters; (3) output lens; (4) photomultiplier tube (H871120); (5) horizontal displacement stage; (6) acquisition system (IQSP480); (7) electric rotating table. wavelength of $652.5 \mathrm{~nm}$ and a bandwidth of $3 \mathrm{~nm}$ should be as close as possible to the input lens. In the design, the filter position is placed at $25 \mathrm{~mm}$ from the input lens, and then the output lens (3) with a focal length of $20 \mathrm{~mm}$ is placed at $25 \mathrm{~mm}$ from the filter; then the output lens focuses the light on the array-PMT (4). The image size of the fiber on the PMT cannot exceed $4 \mathrm{~mm}$ because the photosensitive surface of the PMT detector is $4 \mathrm{~mm} \times 4 \mathrm{~mm}$. When the dimensions of the system parts are determined, the final image size on the PMT is $3 \mathrm{~mm}$. The photomultiplier is a Hamamatsu tube, model H8711-20. If the received power is not enough, the distance between each part can be shortened by changing the horizontal displacement stage (5). At last, an acquisition system (6), a PhotoniQ IQSP480 from Vertilon Corporation, with maximum sampling rate up to $150 \mathrm{kHz}$ transfers the data to a control PC. The center wavelength of the bandpass filters can be changed by rotating the electric rotating table (7) which is similar to that for a single-PMT system, and this will be discussed later.

\section{B. f-FIDA system with single-PMT}

The schematic of the f-FIDA system with multiple singlePMTs is shown in Fig. 3. Each of them consists of two parts. The first part is the optical part. Light from EAST is collected by using an optical fiber with a core diameter of $1500 \mu \mathrm{m}$ and a

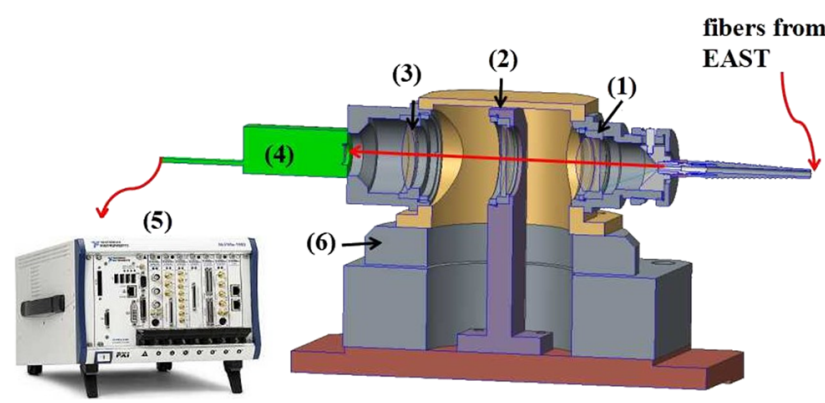

FIG. 3. The schematic of the multi-channel f-FIDA with single-PMT. (1) Input lens; (2) bandpass filters; (3) output lens; (4) photomultiplier tube (H10721-20) with the current amplifier; (5) acquisition system (PXIe 1082 and NI PXIe-6368); (6) rotary table. 


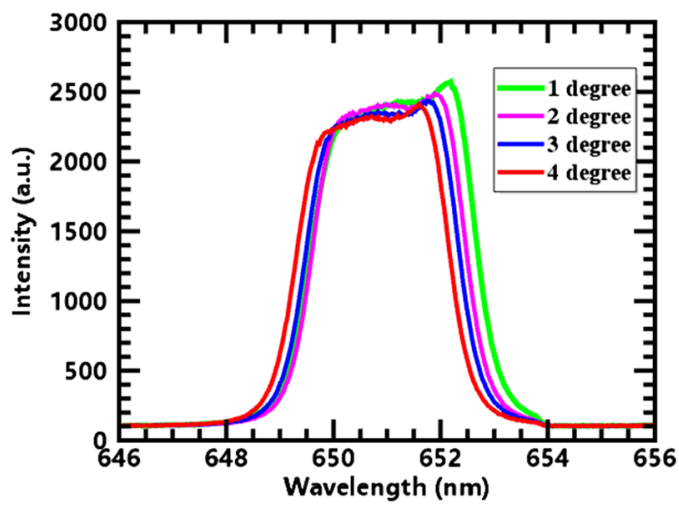

FIG. 4. The measured transmittance curves with different angles between the filter and the optical path. The transmittance curve goes to the shorter wavelength direction when the angle between the filter and the light path is increased.

numerical aperture of 0.37 , then passed through the input lens (1), and focused into parallel rays. The divergence angle at this time is $2^{\circ}$. The input lens has a diameter of $25 \mathrm{~mm}$ and a focal length of $35 \mathrm{~mm}$. Then the OD6 bandpass filters (2) select the desired passband with a center wavelength of $652.5 \mathrm{~nm}$ and a bandwidth of $3 \mathrm{~nm}$. Then the output lens (3) with a diameter of $29 \mathrm{~mm}$ and a focal length of $30 \mathrm{~mm}$ refocuses the light before reaching the PMT. The second part is the integrated part (4), which mainly includes the photomultiplier tube using a Hamamatsu tube (model H10721-20) and the current amplifier. The optical signal of the PMT module is converted into a current signal which is finally converted into a voltage signal by a current amplifier with a bandwidth up to $500 \mathrm{kHz}$. At last, data are collected by using an acquisition system (5), the acquisition box from National Instruments is a NI Pxle-1082, and the acquisition card is NI Pxle-6368. The acquisition card has a bandwidth up to $250 \mathrm{MB} / \mathrm{s}$, and its sampling rate reached $2 \mathrm{MHz}$. Under the premise of ensuring the collimation of the optical path, the focus is on the angle of the light incident on the filter and the change in the transmittance curve of the filter due to this angle. By rotating the electric rotating table (6), the center wavelength of the bandpass filters can change which is measured by single-PMT, as shown in Fig. 4. The transmittance curve goes to the shorter wavelength direction when the angle between the filter and the light path is increased. The center wavelength changes by $0.25 \mathrm{~nm}$ for each $1^{\circ}$, and the result of clockwise rotation and counter clockwise rotation is the same.

\section{PRELIMINARY MEASUREMENTS}

Figure 5 shows waveforms of shot 75439 with $I_{\mathrm{p}} \sim 600 \mathrm{kA}$ and $B_{\mathrm{t}} \sim 2.49 \mathrm{~T}$. NBI1L and NBI1R are injected with a beam energy of $55 \mathrm{keV}$ and beam power of $1.2 \mathrm{MW}$ and $1.5 \mathrm{MW}$, respectively. The diagnostic beam NBI1L is injected for $100 \mathrm{~ms}$ and $10 \%$ duty, and the corresponding time periods for the three blips are 3.2-3.3 s, 4.2-4.3 s, 5.2-5.3 s, as shown in Fig. 5(a). When the beams are turned on, the rate of neutrons increases rapidly and reaches its maximum value, where it remains constant until the beam is turned off, as shown in Fig. 5(b).

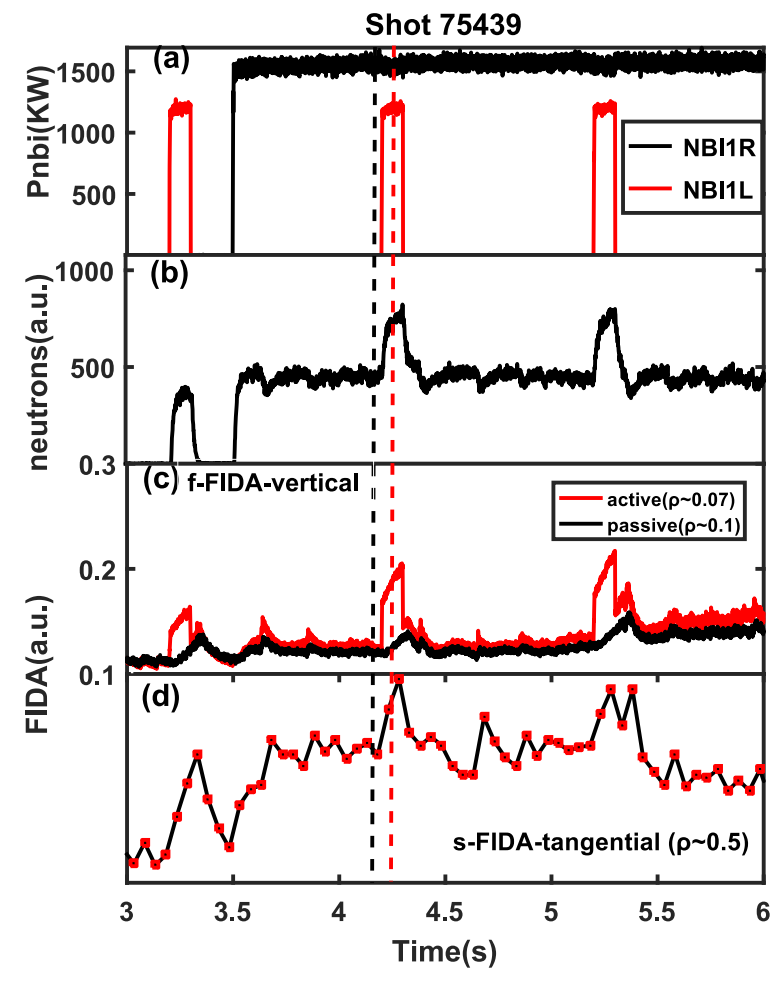

FIG. 5. Time evolution of (a) the heating NBI source, and NBI1L is the diagnostic beam; (b) the signal for neutrons; (c) the raw signals for vertical f-FIDA including active and passive; and (d) the integral signal for tangential s-FIDA between 651 and $654 \mathrm{~nm}$.

There are two methods of background subtraction: One is beam modulation and the other is a paired passive view. Figure 6 shows the typical measured spectrum data from the vertical view during beam-on (red dotted line in Fig. 5) and beam-off (black dotted line in Fig. 5) periods. The beam emission spectrum (BES) between 655 and $657 \mathrm{~nm}$ is strong during the beam-on phase. ${ }^{12}$ The beam emission, $\mathrm{D}_{\alpha}$ light from halo neutrals, and the cold $\mathrm{D}_{\alpha}$ line have been eliminated by an OD3 neutral density filter, and visible bremsstrahlung is a nearly flat spectral feature in this wavelength band. There are several impurity lines present in the blue-shifted wing of the cold $\mathrm{D}_{\alpha}$ line, thus a bandpass filter selects the 651-654 nm range for the application of f-FIDA which avoids the bright sources of light and impurity lines. The background signal is the same in

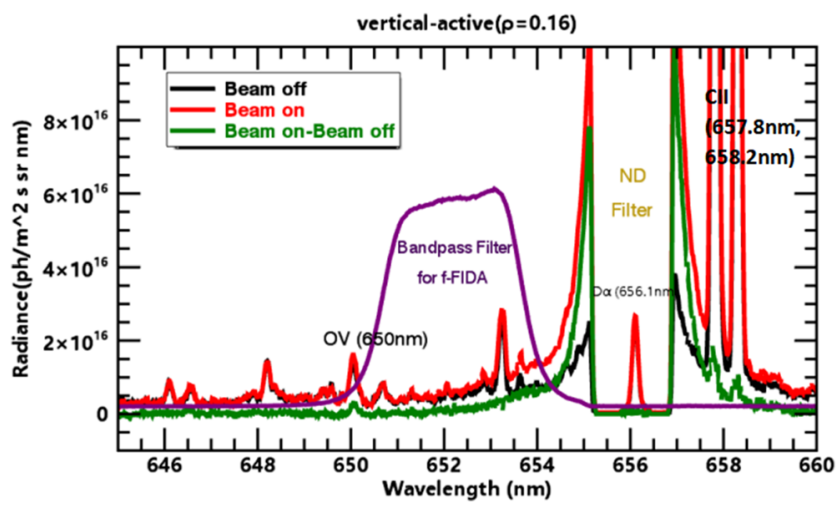

FIG. 6. Measurement of beam-on ( $4.25 \mathrm{~s})$ and beam-off ( $4.19 \mathrm{~s})$ spectra and a transmittance curve of filter on the s-FIDA diagnostic system. 
the beam-on and beam-off periods, as it does not arise from charge exchange and it does not contribute to the measured fast-ion signal. This shows that the plasma is in steady-state. By background subtraction approaches, passive FIDA radiation can be eliminated to obtain net FIDA spectra (green line in Fig. 6). The passive oxygen lines disappear completely.

Figure 5(d) is calculated by integrating the tangential view of s-FIDA spectra signals between 651 and $654 \mathrm{~nm}$ wavelengths. The exposure time of s-FIDA is $20 \mathrm{~ms}$ to ensure that there are two frames in one blip of the NBIs. A clear increase is observed for the beam-on phase and reduction for the beamoff phase, but sometimes when the neutral beam is turned off, the integrated intensity continues to rise, and this is because of the increased background signal of bremsstrahlung.

Figure 5(c) shows the time evolution of two vertical $\mathrm{f}$-FIDA views. One is the vertical active at $\mathrm{R} \sim 1.90 \mathrm{~m}$ and $\rho \sim 0.07$ from B-port viewing the diagnostic beam and the other is the vertical passive at $\mathrm{R} \sim 1.92 \mathrm{~m}$ and $\rho \sim 0.10$ from the N-port. The f-FIDA signals come from two sources: an active component from charge exchange with the injected beam and a passive component from charge exchange with edge neutrals and visible bremsstrahlung. When the diagnostic beam NBI1L is on, the f-FIDA signal with the active view rises and then when the beam turns off there is a rapid drop. During each blip, the active signal should rise as the number of beam ions builds up. The rise depends on the absolute intensity calibration. By contrast, the decay time analysis is independent of the absolute calibration of the f-FIDA diagnostic. The expected behavior of the active signal has features similar to the behavior of the neutron emission, but the rate of neutrons increases rapidly and reaches its maximum value in about $0.1 \mathrm{~s}$ and the active signal is more rapid. The expected rise of the waveform is different for passive f-FIDA than for the neutrons because the weight function has a different energy and pitch dependence actually. This work of passive FIDA has been applied on DIII-D ${ }^{13}$ and NSTX. ${ }^{14}$ The details will be further investigated.

Figure 7 shows the comparison f-FIDA signals between two sets of f-FIDA with single-PMT and array-PMT systems. Figure 7(a) is the zoomed-in image of second of NBI1L shown in Fig. 5(c). Figure 7(b) is the raw active f-FIDA data from the array-PMT at $\mathrm{R} \sim 1.75 \mathrm{~m}$ and $\rho \sim 0.32$ from O-port view in discharge 71695 . EAST plasma data: $I_{\mathrm{p}} \sim 400 \mathrm{kA}, B_{\mathrm{t}} \sim 2.5 \mathrm{~T}$, co-current (NBI1L) and counter-current (NBI2R) neutral beams are injected with a beam voltage of $55 \mathrm{keV}$, and the time period for the second blip of NBI1L is 3.47-3.57 s. Although the onset of the f-FIDA shows a jump in the active signal due to the injected neutral beam, the array-PMT f-FIDA system has a worse signal to noise ratio compared with the single-PMT.

Two f-FIDA systems are currently available, and singlePMT is preferred due to high transmittance, sampling rate, and depth of parallelism. First, after testing, the optical power entering the array-PMT is $15 \mathrm{~mW}$, and the converged optical power is $9 \mathrm{~mW}$, so the optical utilization of the array-PMT is $60 \%$. The same method measures the optical utilization of the single-PMT to $90 \%$. The change in the light utilization depends on the size of the entire system because the size of the array-PMT is fixed and the single-PMT is flexible, mainly
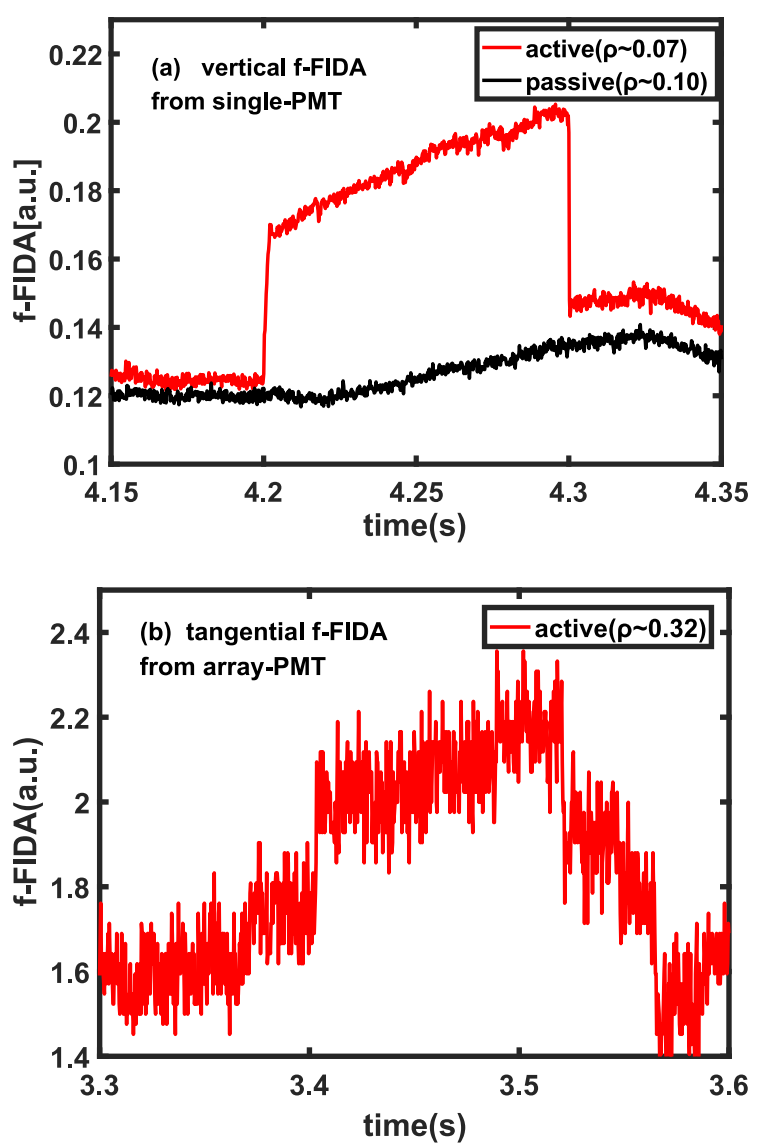

FIG. 7. (a) Details of the f-FIDA signals in Fig. 5(c) between the time ranges of the second blip of NBI1L in discharge 75439. (b) The active f-FIDA signal of the multi-channel f-FIDA with array-PMT in discharge 71695.

depending on the change in the focal length and diameter of the lens. Furthermore, the difference in sampling rates depends on the different acquisition systems. The maximum trigger frequency of the array-PMT is $150 \mathrm{kHz}$ and the single-PMT is $2 \mathrm{MHz}$. Last, due to limited device size of array-PMT, the divergence angle is $5^{\circ}$, but the single-PMT is more flexible which has a bigger lens and a longer focal length, so the depth of parallelism of single-PMT is better than for the array-PMT.

\section{SUMMARY}

To obtain high temporal resolution for FIDA diagnostics, fast filter-based photomultiplier systems have been developed on EAST which can supply the complementary measurements to previous s-FIDA with good spectral resolution measured by high resolution spectrometers. Two sets of f-FIDA with array-PMT and single-PMT will be applied to study the impact of MHD modes and energetic particle instabilities on fastion transport for high performance long pulse discharges on EAST.

\section{ACKNOWLEDGMENTS}

This is supported by the Natural Science Foundation of China under Grant No. 11575249, National Magnetic Confinement Fusion Energy Research Program under Grant 
Nos. 2015GB110005 and 2014GB109004, and Hefei Science Center CAS (No. 2017HSC-IU005).

${ }^{1}$ W. W. Heidbrink, Rev. Sci. Instrum. 81, 10D727 (2010).

${ }^{2}$ W. W. Heidbrink et al., Plasma Phys. Controlled Fusion 46, 1855-1875 (2004).

${ }^{3}$ M. Podestà et al., Rev. Sci. Instrum. 79, 10E521 (2008).

${ }^{4}$ C. M. Muscatello et al., Rev. Sci. Instrum. 81, 10D316 (2010).

${ }^{5}$ M. Salewski et al., Nucl. Fusion 54, 023005 (2014).
${ }^{6}$ M. Osakabe et al., Rev. Sci. Instrum. 79, 10E519 (2008).

${ }^{7}$ B. Geiger et al., Plasma Phys. Controlled Fusion 53, 065010 (2011).

${ }^{8}$ C. Michael et al., Plasma Phys. Controlled Fusion 55, 095007 (2013).

${ }^{9}$ J. Huang et al., Rev. Sci. Instrum. 85, 11E407 (2014).

${ }^{10}$ B. Geiger et al., Plasma Phys. Controlled Fusion 59, 115002 (2017).

${ }^{11}$ Y. M. Hou et al., Rev. Sci. Instrum. 87, 11E552 (2016).

${ }^{12}$ J. Huang et al., Rev. Sci. Instrum. 87, 11E542 (2016).

${ }^{13}$ N. G. Bolte et al., Nucl. Fusion 56, 112023 (2016).

${ }^{14}$ G. Z. Hao et al., Plasma Phys. Controlled Fusion 60, 025026 (2018). 